\title{
Importance of electronic correlations for structural and magnetic properties of the iron pnictide superconductor LaFeAsO
}

\author{
Markus Aichhorn, Leonid Pourovskii and Antoine Georges
}

\section{Linköping University Post Print}

N.B.: When citing this work, cite the original article.

Original Publication:

Markus Aichhorn, Leonid Pourovskii and Antoine Georges, Importance of electronic correlations for structural and magnetic properties of the iron pnictide superconductor LaFeAsO, 2011, Physical Review B. Condensed Matter and Materials Physics, (84), 5, 054529.

http://dx.doi.org/10.1103/PhysRevB.84.054529

Copyright: American Physical Society http://www.aps.org/

Postprint available at: Linköping University Electronic Press http://urn.kb.se/resolve?urn=urn:nbn:se:liu:diva-70217 


\title{
Importance of electronic correlations for structural and magnetic properties of the iron pnictide superconductor LaFeAsO
}

\author{
Markus Aichhorn, ${ }^{1,2}$ Leonid Pourovskii, ${ }^{2,3}$ and Antoine Georges ${ }^{2,4,5}$ \\ ${ }^{1}$ Institute of Theoretical and Computational Physics, TU Graz, Petersgasse 16, Graz, Austria \\ ${ }^{2}$ Centre de Physique Théorique, École Polytechnique, CNRS, F-91128 Palaiseau Cedex, France \\ ${ }^{3}$ Division of Theory and Modeling (IFM), Linköping University, SeRC, SE-581 83 Linköping, Sweden \\ ${ }^{4}$ Collège de France, 11 place Marcelin Berthelot, F-75005 Paris, France \\ ${ }^{5}$ DPMC, Université de Genève, 24 quai E. Ansermet, CH-1211 Genève, Suisse
}

(Received 4 May 2011; published 11 August 2011)

\begin{abstract}
We present calculations of structural and magnetic properties of the iron-pnictide superconductor $\mathrm{LaFeAsO}$ including electron-electron correlations. For this purpose we apply a fully charge self-consistent combination of density-functional theory with the dynamical mean-field theory, allowing for the calculation of total energies. We find that the inclusion of correlation effects gives a good agreement of the arsenic $z$ position with experimental data even in the paramagnetic (high-temperature) phase. Going to low temperatures, we study the formation of the ordered moment in the striped spin-density-wave phase, yielding an ordered moment of about $0.60 \mu_{\mathrm{B}}$, again in good agreement with experiments. This shows that the inclusion of correlation effects improves both structural and magnetic properties of $\mathrm{LaFeAsO}$ at the same time.
\end{abstract}

DOI: $10.1103 /$ PhysRevB.84.054529

PACS number(s): 71.15.Mb, 71.20.Be, 74.70.-b

\section{INTRODUCTION}

Since the discovery of high-temperature superconductivity in iron-based compounds ${ }^{1}$ a lot of research has been dedicated to this fascinating class of materials. On the theoretical side, calculations within density-functional theory (DFT), often performed within the local-density approximation (LDA), could reproduce a variety of properties, but failed in the quantitative description of other features, such as the mass renormalization of the predominately iron- $d$ quasiparticles, which could be improved by the inclusion of correlation effects for the $\mathrm{Fe} 3 d$ electrons..$^{2-5}$ A very puzzling mystery shows up for the compounds exhibiting long-range spin-density-wave (SDW) magnetic ordering at low temperatures. Although the spin-pattern and ordering vectors were well predicted by DFT, it was soon realized that there is a big discrepancy between the magnitude of the measured magnetic moments with theoretical predictions of spin-polarized DFT calculations. For instance, for $\mathrm{LaFeAsO}$, early experimental data pointed to a very small ordered moment in the range of around $0.3 \mu_{\mathrm{B}},{ }^{6,7}$ although recent measurements indicated a somewhat larger moment of $0.63 \mu_{\mathrm{B}}$ in $\mathrm{LaFeAsO}^{8}$ On the other hand, DFT calculations using the experimental crystal structure always gives large values between 1.7 and more than $2 \mu_{\mathrm{B}}{ }^{9-14}$

There is also a strong connection between the value of the ordered moment and details of the crystal structure. As stated above, the ordered moment turns out to be too large in DFT calculations, but in this magnetic case, structural optimization of the $z$ position of the arsenic ions reproduces well the experimental position. ${ }^{12}$ On the other hand, nonmagnetic DFT calculations, which should correspond to the paramagnetic high-temperature phase, gives a too short Fe-As distance with drastic influence on the low-energy electronic structure ${ }^{14}$ Experimentally, the As $z$ position hardly changes across the magnetic transition, ${ }^{6,15}$ a fact that is hard to reconcile within DFT calculations, since the optimized internal structural parameters differ significantly between magnetic and nonmagnetic calculations. The correct description of the equilibrium structure is particularly important for cases where the forces on the ions are important, e.g., phonon calculations.

There were several attempts to improve over simple DFT calculations. Concerning the ordered moment, Yildirim et al. ${ }^{16}$ performed fixed moment DFT calculations in order to study the stability of magnetic ordering patterns. Attempts to include correlation effects by performing LDA $+U$ or GGA $+U$ calculations were not successful. It has been shown ${ }^{17}$ that the magnetic moment even increases with increasing $U$, and even for small interaction values of $U \approx 1 \mathrm{eV}$ the topology of the Fermi surfaces is changed drastically, incompatible with experiments. However, a reduction of the magnetic moment could be found in LDA $+U$ calculation using an effective negative interaction parameter. ${ }^{18}$ Particularly promising are approaches using many-body techniques to include electronic correlation effects. Using a combination of DFT with the dynamical mean-field theory (DMFT) a significant reduction of the magnetic moment could be found for $\mathrm{BaFe} 2 \mathrm{As} 2{ }^{19}$ variational Monte Carlo gave similar results also for other materials, ${ }^{20}$ consistent with a recent comprehensive LDA + DMFT study for a variety of pnictide and chalcogenide materials. ${ }^{21} \mathrm{~A}$ general argument is that quantum fluctuations hinder a large instantaneous iron moment from ordering. ${ }^{19-24}$

Regarding the combination of structural and magnetic properties, one proposal for a better description is to combine in a sophisticated way magnetic and nonmagnetic DFT calculations. ${ }^{25}$ This approach has been used to study the electron-phonon interaction in iron-pnictide superconductors. Improved structural optimization has been performed using a combination of DFT with Gutzwiller wave function techniques, ${ }^{26}$ where the values of the interaction parameters where fitted to give the correct As height above the Fe plane.

The motivation for this paper is to show that the inclusion of correlation effects by LDA + DMFT for the description of $\mathrm{LaFeAsO}$ improves substantially the agreement of both the As $z$ position as well as the ordered magnetic moment between theory and experiment within one set of $a b$ initio calculated 
interaction parameters, which are determined with the constrained random-phase approximation (cRPA). ${ }^{27,28}$ Previous theoretical studies including strong electron-correlations have been focused on the calculation of either magnetic ${ }^{19,21,22}$ or structural properties. $^{26}$

A consistent approach to total-energy calculations and structural optimization within LDA + DMFT (the As $z$ position in $\mathrm{LaFeAsO}$ in the present work) requires selfconsistency over the charge density. LDA + DMFT is often employed within the so-called "one-shot" scheme, where the one-electron part of the Hamiltonian obtained from the bandstructure LDA part is not updated during subsequent DMFT calculations. However, correlation effects will in general induce a certain redistribution of the charge density, which in turn leads to a different Kohn-Sham potential and one-particle part of the Hamiltonian. The correlation-induced changes in the charge density and one-electron potential will also affect the electron-nuclei, Hartree, and exchange-correlation contributions to the LDA + DMFT total energy. Moreover, in some systems (e.g., cerium oxides ${ }^{29}$ ) the charge-density self-consistency has been demonstrated to be important for spectral properties as well.

The paper is organized as follows. In Sec. II we introduce the full charge self-consistent LDA + DMFT method, followed by Sec. III where we present results for the $\mathrm{LaFeAsO}$ system. We draw our conclusions in Sec. IV, which is followed by Appendix A with a more detailed discussion of the influence of the full charge self-consistency on the single-particle spectra.

\section{METHODS}

For the present study, we use a further development of a previously introduced LDA + DMFT implementation, Ref. 5, which is based on the full potential (linearized) augmented plane-wave method as implemented in the WIEN2K package. ${ }^{30}$ Our task of optimizing the arsenic ion position necessitates rather accurate calculations of the total energy, which, as explained in Sec. I, requires a LDA + DMFT scheme fully self-consistent in the charge density. The implementation of full charge self-consistency is currently a topic of high interest, and several schemes have been implemented recently. ${ }^{29,31-33}$

Within the projective technique for formation of the correlated orbitals, Ref. 5, we use the Kohn-Sham (KS) states within a chosen energy window $\mathcal{W}$ to form Wannier-like functions that are treated as correlated orbitals. In the present work, we use an energy window from -6.8 to $2.8 \mathrm{eV}$, spanning the range of Fe- $d$ as well as As- $p$ and O- $p$ states, giving a total number of 22 bands inside the window. On-site interactions were then applied to the five Fe- $d$ orbitals. The very same projection scheme has already been used in Ref. 5. Solving the corresponding single-site quantum impurity problem produces the local self-energy within the correlated orbitals basis set, which is then upfolded into the lattice self-energy $\Sigma_{v v^{\prime}}\left(\mathbf{k}, i \omega_{n}\right)$, where $\omega_{n}$ are Matsubara fermionic frequencies. The lattice self-energy $\hat{\Sigma}\left(\mathbf{k}, i \omega_{n}\right)$ is generally nondiagonal in the subspace of the KS eigenstates $\{v\}(v \in \mathcal{W})$ leading to a nondiagonal lattice Green's function within $\mathcal{W}$ and to the corresponding density matrix:

$$
N_{\nu v^{\prime}}^{k}=\sum_{n} G_{\nu \nu^{\prime}}\left(\mathbf{k}, i \omega_{n}\right) e^{i \omega_{n} 0^{+}}
$$

being also nondiagonal. The charge-density distribution in the real space is then calculated from the density matrix $N_{v v^{\prime}}^{k}$ as follows:

$$
\rho_{\mathrm{DMFT}}(\mathbf{r})=\rho_{o w}(\mathbf{r})+\sum_{k, v v^{\prime}}\left\langle\mathbf{r} \mid \Psi_{k \nu}\right\rangle N_{v v^{\prime}}^{k}\left\langle\Psi_{k v^{\prime}} \mid \mathbf{r}\right\rangle,
$$

where $\Psi_{k v}$ are the KS eigenstates within the energy window $\mathcal{W}, \rho_{\text {ow }}(\mathbf{r})$ is the contribution from states outside $\mathcal{W}$. By substituting into Eq. (2) the expansion of the KS eigenstates within the linear augmented plane-wave (LAPW) basis set one derives formulas for the charge density within the muffin-tin (MT) spheres and in the interstitial. These formulas are generalizations of the standard LAPW expressions to the case of a density-matrix nondiagonal in the space of KS states. As in the standard case, the charge density within the MT spheres is expressed through radial solutions (and their energy derivatives) of the corresponding Schrödinger equation. In the interstitial it is expressed through plane waves. The derivation and relevant formulas for each case are given in Appendix B.

The LDA + DMFT total energy reads ${ }^{34}$

$$
\begin{aligned}
E= & E_{\mathrm{kin}}+E_{c}\left[\rho_{\mathrm{DMFT}}\right]+E_{H}\left[\rho_{\mathrm{DMFT}}\right] \\
& +E_{\mathrm{xc}}\left[\rho_{\mathrm{DMFT}}\right]+\left\langle H_{U}\right\rangle-E_{\mathrm{DC}},
\end{aligned}
$$

where the corresponding contributions in the right-hand side are the kinetic, crystal (electron-nuclei and nuclei-nuclei), Hartree, exchange-correlation, Hubbard, and double-counting correction terms, respectively. The second, third, and fourth terms are evaluated in accordance to the standard DFT-LDA expressions but with the updated LDA + DMFT charge density (2). The kinetic-energy contribution reads

$$
E_{\text {kin }}=E_{\text {band }}-\int d \mathbf{r} v_{\mathrm{KS}}(\mathbf{r}) \rho_{\mathrm{DMFT}}(\mathbf{r}),
$$

where the Kohn-Sham potential $v_{\mathrm{KS}}$ corresponds to the LDA + DMFT charge density $\rho_{\text {DMFT }}$ and the band energy contribution $E_{\text {band }}$ is

$$
E_{\text {band }}=E_{\text {band }}^{o w}+\sum_{\mathbf{k}} \operatorname{Tr} \hat{H}_{\mathrm{KS}}^{k} \hat{N}^{k}=E_{\text {band }}^{o w}+\sum_{\mathbf{k} v} \epsilon_{k v} N_{v v}^{k},
$$

where $H_{\mathrm{KS}}^{k}$ is the one-particle (Kohn-Sham) part of the Hamiltonian, $\epsilon_{k v}$ are its eigenstates with $v \in \mathcal{W}$, and $E_{\text {band }}^{o w}$ is the sum over the occupied Kohn-Sham eigenstates lying outside of the window $\mathcal{W}$.

Finally, the Hubbard term $\left\langle H_{U}\right\rangle$ was evaluated in accordance with the Migdal formula $\left\langle H_{U}\right\rangle=\frac{1}{2} \operatorname{Tr}[\Sigma(i \omega) \mathbf{G}(i \omega)]$, where $\Sigma(i \omega)$ and $\mathbf{G}(i \omega)$ are the impurity self-energy and Green function, respectively.

For the solution of the quantum impurity problem we apply the continuous-time quantum Monte Carlo method in the strong-coupling formulation. ${ }^{35}$ Restricting ourselves to density-density interactions only, as in Ref. 5, we are able to perform calculations down to temperatures as low as $T=77 \mathrm{~K}$ with reasonable numerical effort and without further approximations. For the calculation of total energies, high-quality numerical data are necessary. In order to get an estimate of the statistical error on the total energy, we perform several further iterations (order 10) after self-consistency is reached, yielding an estimate for the standard deviation. 
Interaction parameters have been calculated previously within $\mathrm{cRPA},{ }^{5}$ yielding an average Coulomb interaction of $U=2.7 \mathrm{eV}$ and Hund's exchange of $J=0.8 \mathrm{eV}$. For details of this calculation we refer the reader to Refs. 5, 27, and 28.

As mentioned above, a double-counting correction has to be applied in order to subtract the contribution to the correlation energy already included in the LDA. Several forms have been proposed; we will apply the two most common approximations, which are the around-mean-field (AMF) and the full-localized-limit (FLL) forms,

$$
\begin{gathered}
\Sigma_{\mathrm{DC}}^{\sigma, \mathrm{AMF}}=U(N-n)-J\left(N_{\sigma}-n\right), \\
\Sigma_{\mathrm{DC}}^{\sigma, \mathrm{FLL}}=U(N-0.5)-J\left(N_{\sigma}-0.5\right),
\end{gathered}
$$

where $N$ is the total electronic charge of the impurity problem, $N_{\sigma}$ is its spin-dependent value, and $n$ is the charge per spin and orbital. For the corresponding double-counting energies one can find ${ }^{36}$

$$
\begin{gathered}
E_{\mathrm{DC}}^{\mathrm{AMF}}=\frac{1}{2} U N^{2}-\frac{U+2 l J}{2 l+1} \frac{1}{2} \sum_{\sigma} N_{\sigma}^{2}, \\
E_{\mathrm{DC}}^{\mathrm{FLL}}=\frac{1}{2} U N(N-1)-\frac{J}{2} \sum_{\sigma} N_{\sigma}\left(N_{\sigma}-1\right),
\end{gathered}
$$

with $l=2$ the orbital quantum number for $3 d$ electrons.

Since LSDA calculations give a highly polarized state, we perform our spin-polarized DMFT calculations starting from nonmagnetic LDA calculations.

\section{RESULTS}

Let us start the discussion of our result with the determination of the As $z$ position. We did paramagnetic LDA + DMFT calculations at inverse temperature $\beta=40 \mathrm{eV}^{-1}$, roughly corresponding to room temperature, using the two different types of double-counting corrections mentioned in Sec. II. In Fig. 1 we compare the results with the structure optimization within the LDA, calculated using the WIEN2K

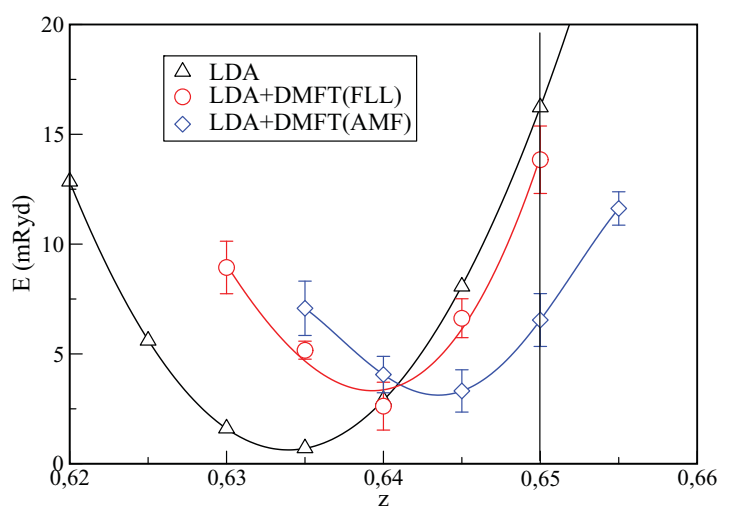

FIG. 1. (Color online) The relative total energy of LaFeAsO as a function of the As height in the unit cell ( $z$ parameter). Solid black line (triangles): LDA result. Red line (circles): LDA + DMFT using FLL double counting. Blue line (diamonds): LDA + DMFT using AMF double counting. Curves are shifted to give similar absolute value of the total energy. Vertical dashed line marks the experimental $z$ position. Error bars are calculated from averaging several further iterations at the self-consistent solution. package. It is obvious from these curves that the inclusion of correlation effects via the DMFT significantly improves over the LDA results. The ion is pushed away from the iron layers toward the experimentally realized $z$ position. Interestingly, the choice of the double-counting correction, although having almost no effect on the single-particle spectra (see below, Sec. IV), has some visible effect on the total energy. This is most likely due to the very small energy scales that one has to deal with in these structure optimizations, where already tiny differences can visibly show up. Nevertheless, using the AMF double counting, the As $z$ position as determined in LDA calculations $(z \approx 0.634)$ is corrected to around $z=0.643$, which has to be compared with the experimental value of $z=0.651$. We attribute the larger distance of the As ion from the iron plane to the fact that in DMFT calculations the ground state of the iron atom is the $S=2$ high spin state, having slightly larger ionic radius then the nonmagnetic state realized in nonmagnetic LDA calculations.

The small discrepancy that we still see between our calculated $z$ values and experimental data is most likely due to the neglection of Coulomb interactions between the iron and arsenic ions ( $p$ - $d$ interactions). It is a very common feature of LDA calculations that the gap between valence bands and ligand bands is too small. Comparing calculated band structure of $\mathrm{LaFeAsO}$ with PES experiments, the discrepancy is about $1 \mathrm{eV}^{37}$ In one-shot LDA + DMFT calculations this gap can artificially be influenced by manually chosen double-counting corrections, which is not the case for full self-consistent calculations (see below, Sec. IV). The correction of this gap would only be possible by the explicit inclusion of $p-d$ interactions, giving also a repulsion between iron and arsenic ions. However, these interactions, without further approximations, go well beyond single-site DMFT calculations as used here.

Having established the improved description of the crystal structure, we move on to magnetic properties of $\mathrm{LaFeAsO}$. From now on, we always use the AMF double-counting correction, meant to be more appropriate for metallic systems. We use here again the experimental value for the As $z$ position in order to compare more directly to experiments. In Fig. 2 we plot the local susceptibility as function of imaginary time for different temperatures. Integrating over imaginary time gives the static susceptibility, which is plotted in the lower panel of Fig. 2 as a black line (open symbols). We see only a very weak dependence on temperature, consistent with experiments. ${ }^{1,15,38}$ Also, the value of $\chi(T)$ is substantially enhanced compared to free electrons, in agreement with Ref. 22, a situation often called "enhanced" Pauli-magnetism. Please note that the upturn at the magnetic-phase transition in experiments is missed here, since here we do calculations only in the paramagnetic phase.

The instantaneous magnetic moment (equal-time correlation function) is large, roughly $1.95 \mu_{\mathrm{B}}$. However, when looking at the ordered moment at low temperatures, the situation is different. To study the ordered moment at low temperatures, we performed LDA + DMFT calculations allowing for spin polarization at $T=116 \mathrm{~K}$, which is well below the magnetic transition temperature of $T_{N} \approx 140 \mathrm{~K}$. As magnetic order pattern we assumed the stripe SDW pattern, 

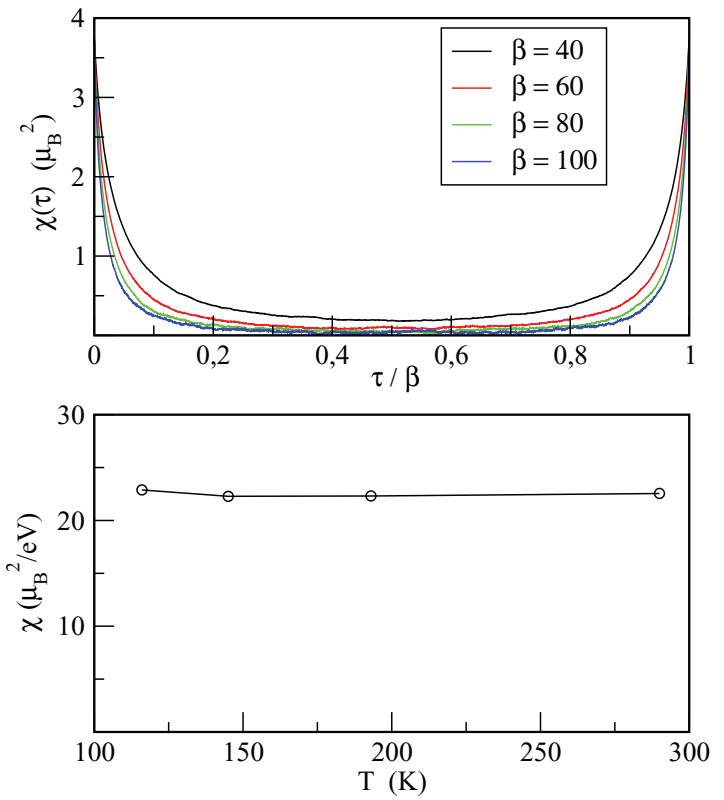

FIG. 2. (Color online) Magnetic properties of $\mathrm{LaFeAsO}$ in the paramagnetic (high-temperature) phase, calculated using AMF double-counting corrections. Upper panel: Local spin susceptibility for inverse temperatures (from top to bottom) $\beta=40,60,80$, and $100 \mathrm{eV}^{-1}$, corresponding to temperatures $T=290,193,145$, and 116 K. Lower panel: Static susceptibility.

as suggested by LSDA calculations as well as experiments. In order to keep calculations as feasible as possible, we used ferromagnetic instead of antiferromagnetic stacking in the $c$ direction, but since the distance of the iron layers is very large, this approximation is well justified. We used the orthorhombic low-temperature unit cell as given in Ref. 6, with ferromagnetic chains running along the short bonds in the $x y$ plane. Doing so, we find an ordered moment of $m=0.58 \mu_{\mathrm{B}}$, significantly smaller than our LSDA value of $m=1.74 \mu_{\mathrm{B}}$. The value of the magnetic ordered moment is almost converged in temperature, since calculations for $T=77 \mathrm{~K}$ give only slightly larger moments of $m=0.60 \mu_{\mathrm{B}}$. In a recent comprehensive LDA + DMFT study a value of $m=0.8 \mu_{\mathrm{B}}$ was reported for $\mathrm{LaFeAsO},{ }^{21}$ the difference in the two results coming from the larger interaction values $U=5.0$ and $J=0.7$ used in Ref. 21 (an estimate on the variation of the magnetic moment as a function of parameters has been given in Ref. 19). For comparison, in the first LDA + DMFT study of the ordered magnetic moment, ${ }^{19}$ done for $\mathrm{BaFe}_{2} \mathrm{As}_{2}$, a similar reduction of the magnetic moment to $m \approx 0.9 \mu_{\mathrm{B}}$ has been found. The temporal fluctuations, which are very strong in the $\mathrm{LaFeAsO}$ compound due to its quite itinerant nature, hinder the instantaneous moments from complete ordering, leaving only a fraction of the moment in the ordered state. Similar arguments have been given for the reduction of the moment in Ref. 19.

Our findings are in qualitative agreement with a recent study on the quenching of the magnetic moment. ${ }^{22}$ However, the former study has been done in the paramagnetic phase, focusing on the influence of local quantum fluctuations on the local moment. A direct comparison of the values of magnetic moments is therefore not appropriate.

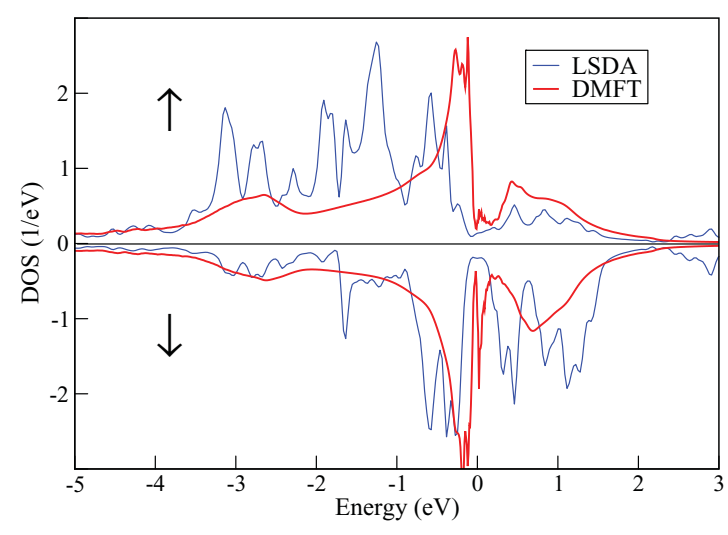

FIG. 3. (Color online) Spin dependent local density of states in the antiferromagnetic phase. Blue thin lines: LSDA. Red thicker lines: LDA + DMFT using AMF double-counting correction.

The reduction of the ordered moment can also be seen in the local density of states as shown in Fig. 3, where we plot the momentum integrated spectral function for the $\mathrm{Fe} 3 d$ electrons. Real frequency data have been obtained by using the stochastic maximum entropy method. ${ }^{39}$ In LSDA the splitting between majority and minority spins is large, whereas we see only a small gap in the LDA + DMFT spectra due to the smaller moment.

\section{CONCLUSIONS}

In this paper, we presented an extension of the previously introduced LDA + DMFT approach ${ }^{5}$ based on the augmented linearized plane-wave basis to full charge self-consistency, and applied this approach to structural and magnetic properties of the iron superconductor $\mathrm{LaFeAsO}$. We calculated the total energy as a function of the pnictogen height, and found that the inclusion of correlation effects shift the minimum position from $z=0.632$ to roughly $z=0.644$, a much better agreement with the experimental value of $z=0.651$. This increased distance of the As ion from the Fe plane is due to the high spin state of iron, which is formed due to local interactions.

Considering the magnetic properties, we calculated the local spin susceptibility and found that it shows very weak temperature dependence in the paramagnetic state, in accordance with enhanced Pauli magnetism. In the low- $T$ SDW phase, we calculated the ordered moment in the stripelike antiferromagnetic phase, and found a moment of $m \approx 0.6 \mu_{\mathrm{B}}$, in much better agreement with experimental values than the LSDA value, which can be (for the experimental crystal structure) as high as $2 \mu_{\mathrm{B}}$.

In summary, the inclusion of correlation effects significantly improve both structural and magnetic properties of $\mathrm{LaFeAsO}$ within one set of parameters. This strongly points to the importance of local quantum fluctuations and correlations for the physics of iron-based superconductors.

\section{ACKNOWLEDGMENTS}

We acknowledge fruitful discussions with J. Mravlje, S. Biermann, V. Vildosola, M. Ferrero, and O. Parcollet. This work was supported by IDRIS/GENCI (Project No. 101393). M.A. gratefully acknowledges financial support from 


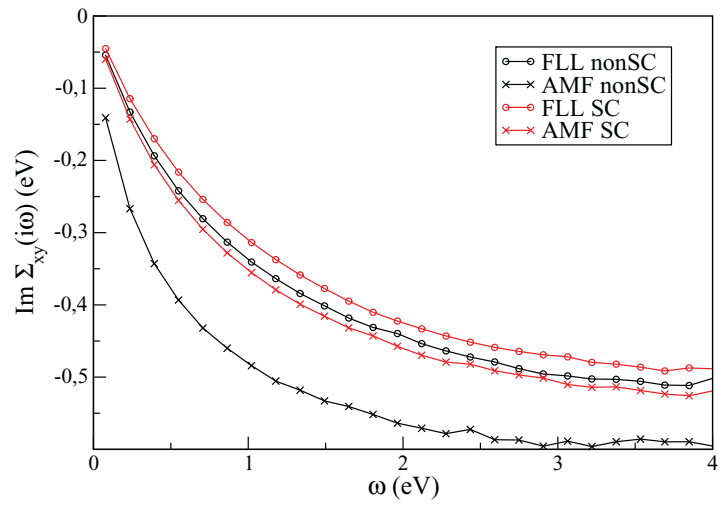

FIG. 4. (Color online) Comparison of the self-energies for the $d_{x y}$ orbital for low Matsubara frequencies at $\beta=40 \mathrm{eV}^{-1}$. Dashed lines: one-shot non-self-consistent calculations. Solid lines: self-consistent calculations. Circles: FLL double counting. Crosses: AMF double counting.

the Austrian Science Fund (Projects No. J2760, No. F4103, and No. P18551). L.P. acknowledges financial support of Linköping Linnaeus Initiative for Novel Functional Materials (LiLi-NFM) and Swedish Research Council (VR) as well as computational resources provided by the Swedish National Infrastructure for Computing (SNIC). A.G. acknowledges the support of the Agence Nationale de la Recherche (under the grant "PNICTIDES" ANR 2010 BLAN 0408 04) and the hospitality of the Université de Genève (with support from the MANEP program).

\section{APPENDIX A: SINGLE-SHOT VERSUS FULL CHARGE SELF-CONSISTENCY}

In this appendix we show that self-consistent calculations improve over one-shot calculations regarding the choice of the double-counting correction. The most straightforward quantity to look at is the impurity self-energy on the Matsubara axis, which is not affected by any analytic continuation problem to real frequencies. Fig. 4 shows the result for the iron $d_{x y}$ orbital. It is obvious that non-self-consistent calculations give a sizable discrepancy between FLL and AMF double counting, which is largely canceled in self-consistent calculations. From this plot we can also see that the FLL one-shot calculation is in better agreement with the self-consistent calculations, whereas AMF is far off.

Going to the real axis, we can look at the momentum integrated spectral function, and compare it with its LDA result, shown in Fig. 5. Again, similar as discussed above, the agreement between different calculations is much better in the self-consistent case (lower panel), and one-shot FLL is again in better agreement. A striking difference between non-self-consistent and self-consistent calculations is that there is no spurious shift of the As and $\mathrm{O} p$ states due to the approximate nature of the double-counting correction. Both calculations show the features largely related to $\mathrm{As}$ and $\mathrm{O}$ at basically the same energy as in the LDA calculation. As a result, it is not easily possible in self-consistent calculations to use a manually adjusted double-counting correction for increasing or decreasing the $p-d$ gap.

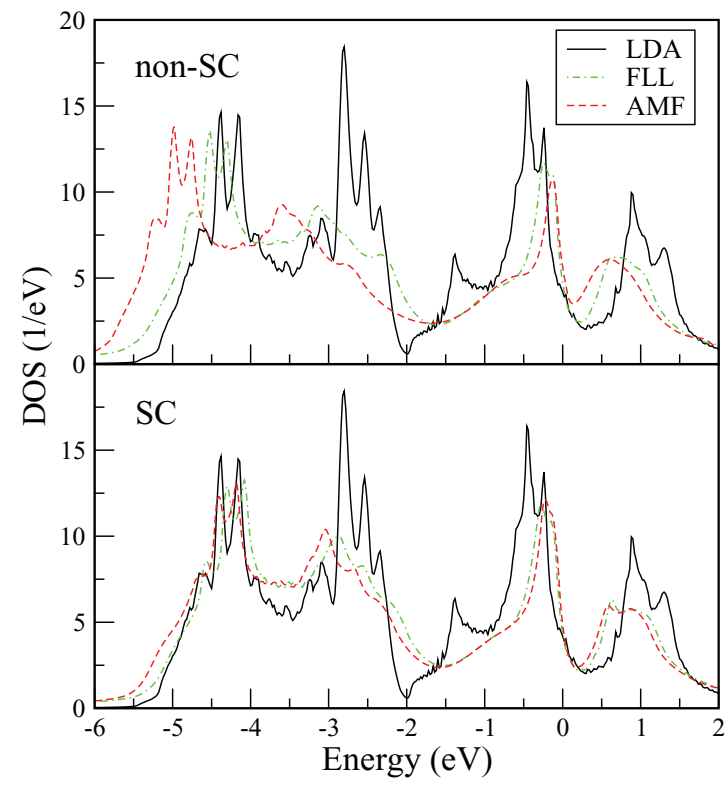

FIG. 5. (Color online) Comparison of the $k$-summed spectral function for different LDA + DMFT calculations. Black solid lines: LDA result. Green dot-dashed: LDA + DMFT, FLL. Red dashed: LDA + DMFT, AMF. Upper panel: non-self-consistent. Lower panel: self-consistent calculations.

\section{APPENDIX B: LDA + DMFT CHARGE DENSITY WITHIN THE (L)APW BASIS SET}

\section{Charge density within MT spheres}

In the LAPW framework the basis functions within an MT sphere $\alpha$ are the radial solutions $u_{l}^{\alpha, \sigma}$ of the Schrödinger equation labeled by the orbital quantum number $l$ and spin $\sigma$ and evaluated at a certain linearization energy $E_{l 1}^{\alpha}$ and their corresponding energy derivatives $\dot{u}_{l}^{\alpha, \sigma}$ evaluated at the same energy. Additional radial solutions can be introduced to account for semicore states; they are evaluated at a corresponding energy $E_{l 2}^{\alpha}$ of the semicore band. The angular and spin dependence for the solutions within the MT spheres is given by corresponding spherical harmonics $Y_{m}^{l}(\hat{r})$ and spinors $\chi_{\sigma}$. The functions $u_{l}^{\alpha, \sigma}\left(E_{l 1}^{\alpha}\right) Y_{m}^{l} \chi_{\sigma}, \dot{u}_{l}^{\alpha, \sigma}\left(E_{1 l}^{\alpha} Y_{m}^{l} \chi_{\sigma}\right.$ for valence and $u_{l}^{\alpha, \sigma}\left(E_{2 l}^{\alpha}\right) Y_{m}^{l} \chi_{\sigma}$ for semicore states will contribute to a given eigenvector $\Psi_{k v}$ with the corresponding coefficients $A_{l m}^{\nu, \alpha}(\mathbf{k}, \sigma), B_{l m}^{\nu, \alpha}(\mathbf{k}, \sigma)$ and $C_{l m}^{v, \alpha}(\mathbf{k}, \sigma)$, respectively, as defined in Ref. 5.

Let us designate the set of these basis functions $\left\{u_{l}^{\alpha, \sigma}\left(E_{1 l}^{\alpha}\right) Y_{m}^{l} \chi_{\sigma}, \dot{u}_{l}^{\alpha, \sigma}\left(E_{1 l}^{\alpha}\right) Y_{m}^{l} \chi_{\sigma}, u_{l}^{\alpha, \sigma}\left(E_{2 l}^{\alpha}\right) Y_{m}^{l} \chi_{\sigma}\right\}$ for a given MT sphere $\alpha$ and quantum numbers $l, m, \sigma$ as $x_{l}^{\alpha, \sigma} Y_{m}^{l} \chi_{\sigma}$ and the set of corresponding coefficients $\left\{A_{l m}^{v, \alpha}(\mathbf{k}, \sigma), B_{l m}^{v, \alpha}(\mathbf{k}, \sigma), C_{l m}^{v, \alpha}(\mathbf{k}, \sigma)\right\}$ with which they contribute to a given eigenvector $\Psi_{k v}$ as $S_{l m}^{v, \alpha}(\mathbf{k}, \sigma)$. Hence within a given MT sphere the KS eigenvector $\Psi_{k v}^{\sigma}(\mathbf{r})$ is expanded as $\sum_{l m i} S_{l m i}^{\nu, \alpha}(\mathbf{k}, \sigma) x_{l i}^{\alpha, \sigma}(r) Y_{m}^{l}(\hat{r}) \chi_{\sigma}$, where $i$ runs over all radial functions $\{x\}$ and corresponding coefficients $\{S\}$.

Using those designations, the charge-density contribution from the states within the energy window $\mathcal{W}$ [the second term in right-hand side of Eq. (2)] can be rewritten for a given MT 
sphere $\alpha$ and for a given spin as

$$
\begin{aligned}
\rho_{\sigma \alpha}^{W}(\mathbf{r})= & \sum_{k} \sum_{l l^{\prime}} \sum_{i j} x_{l i}^{\alpha \sigma}(r) x_{l^{\prime} j}^{\alpha \sigma}(r) \\
& \times \sum_{m m^{\prime}} Y_{m}^{l}(\hat{r})\left(Y_{m^{\prime}}^{l^{\prime}}(\hat{r})\right)^{*} \\
& \times \sum_{\nu \nu^{\prime} \in \mathcal{W}} S_{l m i}^{\nu, \alpha}(\mathbf{k}, \sigma)\left(S_{l^{\prime} m^{\prime} j}^{\nu^{\prime}, \alpha}(\mathbf{k}, \sigma)\right)^{*} N_{\nu \nu^{\prime}}^{\mathbf{k}} .
\end{aligned}
$$

To represent the angular dependence of the charge density it is expanded, within a given MT sphere, in real spherical harmonics $Y_{m}^{R l}(\hat{r})$,

$$
\rho_{\sigma \alpha}^{\mathcal{W}}(\mathbf{r})=\sum_{l m} \rho_{\sigma \alpha}^{l m}(r) Y_{m}^{R l}(\hat{r})
$$

For $\quad \rho_{\sigma \alpha}^{l m}(r)=\int d \hat{r} \rho_{\sigma \alpha}^{\mathcal{W}}(\mathbf{r}) Y_{m}^{R l}(\hat{r}) \quad$ one obtains from Eq. (B1)

$$
\begin{aligned}
\rho_{\sigma \alpha}^{l_{1} m_{1}}(r)= & \sum_{k} \sum_{l l^{\prime}} \sum_{i j} x_{l i}^{\alpha \sigma}(r) x_{l^{\prime} j}^{\alpha \sigma}(r) \sum_{m m^{\prime}} C_{l m l_{1} m_{1}}^{l^{\prime} m^{\prime}} \\
& \times \sum_{\nu \nu^{\prime} \in \mathcal{W}} S_{l m i}^{\nu, \alpha}(\mathbf{k}, \sigma)\left(S_{l^{\prime} m^{\prime} j}^{\nu^{\prime}, \alpha}(\mathbf{k}, \sigma)\right)^{*} N_{\nu \nu^{\prime}}^{\mathbf{k}},
\end{aligned}
$$

where $C_{l m l_{1} m_{1}}^{l^{\prime} m^{\prime}}=\int d \Omega Y_{m}^{l}(\Omega)\left[Y_{m^{\prime}}^{l^{\prime}}(\Omega)\right]^{*} Y_{m_{1}}^{R l_{1}}(\Omega)$ are the corresponding Gaunt coefficients.

\section{Charge density in the interstitial region}

In the interstitial region the LAPW basis functions are plane waves $\frac{1}{\sqrt{V}} e^{i(\mathbf{k}+\mathbf{G}) \mathbf{r}}$, where $G$ is the reciprocallattice vector and $V$ is the unit-cell volume, contributing to a given KS eigenvector $\left|\Psi_{k \nu}^{\sigma}\right\rangle$ with the corresponding coefficients $a_{G}^{v}(\mathbf{k}, \sigma)$. Substituting this into
Eq. (2) one obtains the contribution of the states within the energy window $\mathcal{W}$ to the charge density in the interstitial:

$$
\begin{aligned}
\rho_{I}^{\mathcal{W}}(\mathbf{r})= & \frac{1}{V} \sum_{\mathbf{k}} \sum_{\nu \nu^{\prime} \in \mathcal{W}}\left[\sum_{G} a_{G}^{\nu}(\mathbf{k}, \sigma) e^{i \mathbf{k}_{\mathbf{G}} \mathbf{r}}\right. \\
& \left.\times \sum_{G^{\prime}}\left(a_{G^{\prime}}^{\nu^{\prime}}(\mathbf{k}, \sigma)\right)^{*} e^{-i \mathbf{k}_{\mathbf{G}^{\prime}} \mathbf{r}}\right] N_{\nu \nu^{\prime}}^{\mathbf{k}} \\
= & \frac{1}{V} \sum_{\mathbf{k}} \sum_{\nu \nu^{\prime} \in \mathcal{W}}\left[\sum_{G} a_{G}^{\nu}(\mathbf{k}, \sigma) e^{i \mathbf{G r}}\right. \\
& \left.\times \sum_{G^{\prime}}\left(a_{G^{\prime}}^{\nu^{\prime}}(\mathbf{k}, \sigma)\right)^{*} e^{-i \mathbf{G}^{\prime} \mathbf{r}}\right] N_{\nu v^{\prime}}^{\mathbf{k}},
\end{aligned}
$$

where $\mathbf{k}_{\mathbf{G}}=\mathbf{k}+\mathbf{G}$.

In the actual computation of Eq. (B4) one may transform the interstitial wave function $\sum_{G} a_{G}^{\nu}(\mathbf{k}, \sigma) e^{i \mathbf{G r}}$ to a auxiliary mesh in the real space via the fast Fourier transform:

$$
b_{\mathbf{R}}^{v}(\mathbf{k}, \sigma)=\sum_{G} a_{G}^{v}(\mathbf{k}, \sigma) e^{i \mathbf{G R}},
$$

therefore getting rid of the double sum over $\mathbf{G}$ and $\mathbf{G}^{\prime}$ in Eq. (B4). The charge density on the axillary mesh then reads

$$
\rho^{I}(\mathbf{R})=\frac{1}{V} \sum_{\mathbf{k}} \sum_{\nu \nu^{\prime} \in \mathcal{W}} b_{\mathbf{R}}^{\nu}(\mathbf{k}, \sigma)\left[b_{\mathbf{R}}^{v^{\prime}}(\mathbf{k}, \sigma)\right]^{*} N_{\nu \nu^{\prime}}^{\mathbf{k}},
$$

which is then again transformed back to the reciprocal space via inverse fast Fourier transform:

$$
\rho^{I}(\mathbf{G})=\sum_{R} \rho_{\mathbf{R}}^{I} e^{-i \mathbf{G R}} .
$$

${ }^{1}$ Y. Kamihara, T. Watanabe, M. Hirano, and H. Hosono, J. Am. Chem. Soc. 130, 3296 (2008).

${ }^{2}$ K. Haule, J. H. Shim, and G. Kotliar, Phys. Rev. Lett. 100, 226402 (2008).

${ }^{3}$ K. Haule and G. Kotliar, New J. Phys. 11, 025021 (2009).

${ }^{4}$ V. I. Anisimov, D. M. Korotin, M. A. Korotin, A. V. Kozhevnikov, J. Kunes, A. O. Shorikov, S. L. Skornyakov, and S. V. Streltsov, J. Phys.: Condens. Matter 21, 075602 (2009).

${ }^{5}$ M. Aichhorn, L. Pourovskii, V. Vildosola, M. Ferrero, O. Parcollet, T. Miyake, A. Georges, and S. Biermann, Phys. Rev. B 80, 085101 (2009).

${ }^{6}$ C. de La Cruz et al., Nature (London) 453, 899 (2008).

${ }^{7}$ M. A. McGuire et al., Phys. Rev. B 78, 094517 (2008).

${ }^{8}$ N. Qureshi, Y. Drees, J. Werner, S. Wurmehl, C. Hess, R. Klingeler,

B. Buchner, M. T. Fernandez-Diaz, and M. Braden, Phys. Rev. B 82, 184521 (2010).

${ }^{9}$ J. Dong et al., Europhys. Lett. 83, 27006 (2008).

${ }^{10}$ C. Cao, P. J. Hirschfeld, and H.-P. Cheng, Phys. Rev. B 77, 220506 (2008).

${ }^{11}$ F. Ma and Z.-Y. Lu, Phys. Rev. B 78, 033111 (2008).

${ }^{12}$ I. I. Mazin, M. D. Johannes, L. Boeri, K. Koepernik, and D. J. Singh, Phys. Rev. B 78, 085104 (2008).
${ }^{13}$ S. Ishibashi, K. Terakura, and H. Hosono, J. Phys. Soc. Jpn. 77, 053709 (2008).

${ }^{14}$ Z. P. Yin, S. Lebègue, M. J. Han, B. P. Neal, S. Y. Savrasov, and W. E. Pickett, Phys. Rev. Lett. 101, 047001 (2008).

${ }^{15}$ T. Nomura, S. W. Kim, Y. Kamihara, M. Hirano, P. V. Sushko, K. Kato, M. Takata, A. L. Shluger, and H. Hosono, Supercond. Sci. Technol. 21, 125028 (2008).

${ }^{16}$ T. Yildirim, Phys. Rev. Lett. 101, 057010 (2008).

${ }^{17}$ S. Ishibashi and K. Terakura, J. Phys. Soc. Jpn. Suppl. C 77, 91 (2008).

${ }^{18}$ J. Ferber, Y.-Z. Zhang, H. O. Jeschke, and R. Valentí, Phys. Rev. B 82, 165102 (2010).

${ }^{19}$ Z. P. Yin, K. Haule, and G. Kotliar, Nat. Phys. 7, 294 (2011).

${ }^{20}$ T. Misawa, K. Nakamura, and M. Imada, J. Phys. Soc. Jpn. 80, 023704 (2011)

${ }^{21}$ Z. P. Yin, K. Haule, and G. Kotliar, e-print arXiv:1104.3454 (to be published).

${ }^{22}$ P. Hansmann, R. Arita, A. Toschi, S. Sakai, G. Sangiovanni, and K. Held, Phys. Rev. Lett. 104, 197002 (2010).

${ }^{23}$ H. Lee, Y.-Z. Zhang, H. O. Jeschke, and R. Valentí, Phys. Rev. B 81, 220506 (2010). 
${ }^{24}$ Y.-Z. Zhang, H. Lee, I. Opahle, H. O. Jeschke, and R. Valenti, J. Phys. Chem. Solids 72, 324 (2011).

${ }^{25}$ L. Boeri, M. Calandra, I. I. Mazin, O. V. Dolgov, and F. Mauri, Phys. Rev. B 82, 020506 (2010).

${ }^{26}$ G. T. Wang, Y. Qian, G. Xu, X. Dai, and Z. Fang, Phys. Rev. Lett. 104, 047002 (2010).

${ }^{27}$ T. Miyake, K. Nakamura, R. Arita, and M. Imada, J. Phys. Soc. Jpn. 79, 044705 (2010).

${ }^{28}$ T. Miyake, L. Pourovskii, V. Vildosola, S. Biermann, and A. Georges, J. Phys. Soc. Jpn. Suppl. C 77, 99 (2008).

${ }^{29}$ L. V. Pourovskii, B. Amadon, S. Biermann, and A. Georges, Phys. Rev. B 76, 235101 (2007).

${ }^{30}$ P. Blaha, K. Schwarz, G. Madsen, D. Kvasnicka, and J. Luitz, WIEN2k, An Augmented Plane Wave + Local Orbitals Program for Calculating Crystal Properties (Techn. Universitat Wien, Austria, 2001).
${ }^{31}$ S. Y. Savrasov and G. Kotliar, Phys. Rev. B 69, 245101 (2004).

${ }^{32}$ K. Haule, C.-H. Yee, and K. Kim, Phys. Rev. B 81, 195107 (2010).

${ }^{33}$ B. Amadon, Phys. Rev. Lett. 104, 233601 (2010).

${ }^{34}$ B. Amadon, S. Biermann, A. Georges, and F. Aryasetiawan, Phys. Rev. Lett. 96, 066402 (2006).

${ }^{35}$ P. Werner, A. Comanac, L. de' Medici, M. Troyer, and A. J. Millis, Phys. Rev. Lett. 97, 076405 (2006).

${ }^{36}$ E. R. Ylvisaker, W. E. Pickett, and K. Koepernik, Phys. Rev. B 79, 035103 (2009).

${ }^{37}$ W. Malaeb et al., J. Phys. Soc. Jpn. 77, 093714 (2008).

${ }^{38}$ D. J. Singh and M.-H. Du, Phys. Rev. Lett. 100, 237003 (2008).

${ }^{39}$ K. S. D. Beach, e-print arXiv:cond-mat/0403055 (to be published). 